\title{
Link between optimization and local stability of a low-dissipation heat engine: Dynamic and energetic behaviors
}

\author{
J. Gonzalez-Ayala, ${ }^{1,2,{ }^{*}}$ M. Santillán, ${ }^{3, \dagger}$ I. Reyes-Ramírez, ${ }^{4, \dagger}$ and A. Calvo-Hernández ${ }^{1,2, \S}$ \\ ${ }^{1}$ Departamento de Física Aplicada, Universidad de Salamanca, 37008 Salamanca, Spain \\ ${ }^{2}$ Instituto de Física Fundamental y Matemáticas, Universidad de Salamanca, 37008 Salamanca, Spain \\ ${ }^{3}$ Centro de Investigación y Estudios Avanzados del IPN Unidad Monterrey, 66600 Apodaca, Nuevo León, Mexico \\ ${ }^{4}$ Instituto Politécnico Nacional-UPIITA, 07340 Ciudad de México, Mexico
}

(Received 19 July 2018; published 27 September 2018)

\begin{abstract}
In the present paper we study the connection between local stability and energetic properties in low-dissipation heat engines operating in the maximum-power and maximum-compromise $(\Omega)$ regimes. We consider two different feedback regulatory pathways: (1) one in which restitutive forces linearly depend on the deviations from the stationary values of the heat exchanges with the hot and cold reservoirs and (2) another where restitutive forces depend on the deviations from the stationary values of the power output and the heat outflux into the cold reservoir. The first dynamics leads to an isolated stable point while in the second one the system is metastable. Further analysis of random perturbations from the steady state gives valuable information about the dynamic behavior of thermodynamic properties like entropy, power, and efficiency in both operation regimes.
\end{abstract}

DOI: 10.1103/PhysRevE.98.032142

\section{INTRODUCTION}

Thermodynamic optimization of irreversible heat-engine models has been performed taking into account a variety of objective functions, among them maximum efficiency, maximum power (MP), and trade-off criteria [like the maximum $\Omega$ $(\mathrm{M} \Omega)$ and maximum ecological (ME) functions] [1-9]. The engine energetic properties resulting from these regimes have transcended the specific models they arose from [10-16], and so their validity can be regarded as general, provided certain symmetries or coupling considerations are present. In particular, the efficiency at MP has been extensively studied [17,18], giving rise in the fully symmetric case to the paradigmatic Curzon-Ahlborn efficiency $\eta_{C A}$ [1].

In the present work we focus on the so-called lowdissipation heat engine (LDHE) [3] model. The reason is that, under a small dissipation assumption, it is possible to study operation regimes, like MP and M $\Omega$, without specific information regarding the nature of heat fluxes or dissipation mechanisms. The equivalence between the LDHE and other models such as the minimally nonlinear irreversible heat engine [5,19]; the model by Sheng and Tu [6] describing the coupling of two stationary irreversible fluxes to model a heat engine, using the so-called weighted thermal fluxes; the endoreversible or irreversible Carnot-like heat engine [20,21]; and (under certain circumstances) stochastic heat engines $[22,23]$ has been discussed elsewhere. Optimization in LDHE models is achieved through suitable contact times $[21,24]$ or an equivalent set of variables (such as a partial contact time

\footnotetext{
*jgonzalezayala@usal.es

†msantillan@cinvestav.mx

†ireyesram@hotmail.com

§anca@usal.es
}

and a dimensionless total time) $[25,26]$. An important feature of LDHE models is that, after optimizing them, the relations between the operation regime and variables like power output $P$, efficiency $\eta$, and entropy generation $\Delta S_{\text {tot }}$, as well as the relations among them, are given usually in terms of quite general functions that ultimately depend on the dissipation symmetries. For instance, one can obtain upper and lower bounds for the maximum power efficiency in terms of dissipation symmetries. Furthermore, it is possible to introduce specific time constraints to reproduce behaviors of irreversible and endoreversible heat engines (HEs).

Optimizing the thermodynamic characteristics of an engine is necessary to have an adequate performance, but it may not be sufficient. From a dynamical perspective, it is also important that the engine optimal operation regime is stable. This reasoning is in the same line of thought as the work of Pietzonka and Seifert [27], who proposed that constancy is an additional ingredient in the optimization of heat engines. In LDHEs, stability of the thermodynamically optimal regime of operation means that, after any perturbation on the value of the control parameters (contact times with with thermal baths), they would naturally return to their optimal value. In some of the above quoted examples for which LDHE models are adequate, control parameters are directly and tightly regulated, thus ensuring the system stability. However, it is not possible to ensure that this is always the case. In this regard, the authors of Ref. [28] have emphasized the lack of a systematic and quantitative description of how limited control affects the performance of heat engines. We have argued elsewhere [29] that in many instances control parameters are indirectly regulated via design parameters, like material properties of the system components. In this last scenario random environmental fluctuations would drive the system out from the optimal performance regime, but the system could return to the optimal regime if it is dynamically stable. Taking 
this discussion into account, the present work is advocated at studying the dynamical stability of LDHEs in different regimes of operation. This problem is related to some recent works regarding power fluctuations and large efficiencies (see, for example, Refs. [27,30]).

Since the specific mechanisms that cause heat flows in LDHE models are unknown, it is necessary, for the study of local stability here performed, to introduce ad hoc restitutive forces. In a previous work [29] we tackled this problem for engines operating at maximum power by considering two different scenarios:

(1) Restitutive forces linearly depend on the deviation from the stationary value of the heat exchanged per cycle between the engine and the surrounding temperature baths.

(2) Restitutive forces linearly depend on the deviations from their stationary value of the power output and the heat outflux into the cold heat reservoir.

In both cases we observed that the system thermodynamic and stability properties are affected by the same parameters. This opens a new and complementary perspective on the study of heat devices in the finite time domain. Interestingly, similar results have been obtained for other systems in which the underlying mechanisms are known: irreversible Carnotlike heat engines [31], a Brownian motor model describing functioning of motor proteins [32], and a model for enzymatic reactions that couple exothermic and endothermic reactions [33].

To the best of our knowledge, none of the works dealing with thermodynamic and stability optimization of heat engines has paid attention to the behavior of thermodynamic variables like entropy, efficiency,and power output while the system is perturbed from the steady state. However, it is possible that the trajectories followed by those variables are not arbitrary, but conditioned by thermodynamic considerations. The present work is aimed at studying this possibility and, by doing so, at improving our knowledge of how dissipation symmetries, time constraints, and the evolution of energetic functions are related in LDHEs obeying different optimization criteria: maximum power and maximum $\Omega$ function [24]. The $\Omega$ function optimization criterion, together with the so-called ecological operation regime [2], represents optimal trade-offs between large power output and low entropy production $(\sigma)$. In the ecological operation regime the merit function that is optimized is

$$
E \equiv P-T_{c} \sigma
$$

where $T_{c}$ is the temperature of the cold heat reservoir. On the other hand, the function $\Omega$ was defined in such a way that it represents a compromise between useful energy and energy lost for an arbitrary energy converter. In the case of heat engines, this function is defined as the difference between maximum power gain $\left(P_{\text {gain }}=P-P_{\text {min }}\right)$ and minimum power loss $\left(P_{\text {loss }}=P_{\max }-P\right)$. By taking into consideration that the heat input is $\dot{Q}_{h}$ and that the minimum achievable efficiency is zero, it follows that $\Omega \equiv P_{\text {gain }}-P_{\text {loss }}$ can be written as

$$
\Omega=2 P-P_{\max }-P_{\text {loss }}=\left(2 \eta-\eta_{C}\right) \dot{Q}_{h} .
$$

In order to model the effects of variations on the operation regime due to random external effects, we include a study of random perturbations. This allows us to obtain valuable information regarding the stability and optimized energetics of the heat device.

The paper is organized as follows: The LDHE's MP and $\mathrm{M} \Omega$ regimes are reviewed in Sec. II. In Secs. III and IV we study the stability of both operation regimes when the restitutive forces respectively depend on the heat exchanged with both thermal baths and on the power output and the heat outflux into the cold bath. In Sec. V we study how the system energetic properties behave when the system undergoes random perturbations from the steady state. Finally, in Sec. VI we present some concluding remarks.

\section{MAXIMUM-POWER AND MAXIMUM- $\Omega$ REGIMES}

The so-called low-dissipation heat engine model consists of a Carnot engine with small deviations from the reversible cycle of operation. These deviations are due to dissipations at the isothermal branches, which occur at finite time. The adiabatic processes are considered instantaneous in this model, although the influence of the time in the adiabatic branches of the cycle were discussed in Ref. [34]. Under these approximations, the heat exchanges between the LDHE and the cold $\left(T_{c}\right)$ and hot $\left(T_{h}>T_{c}\right)$ thermal reservoirs can be modeled as first order deviations from the reversible exchanges of a Carnot engine [3]:

$$
\begin{gathered}
Q_{h}=T_{h} \Delta S\left(1-\frac{\Sigma_{h}}{\Delta S t_{h}}\right), \\
Q_{c}=T_{c} \Delta S\left(-1-\frac{\Sigma_{c}}{\Delta S t_{c}}\right),
\end{gathered}
$$

where $\Delta S$ is the reversible entropy change along the $T_{h}$ isothermal process, $\Sigma_{c, h}$ are coefficients in which all the information about the device dissipation intrinsic properties is contained, and $t_{c, h}$ are respectively the contact times with the hot and cold reservoirs. Some specific applications of this approach have been described in Ref. [19], where the dissipation term is related to Joule heating. Furthermore, in Ref. [35], the authors show that the upper bounds for the LDHE efficiency at the maximum-power and maximum ecological regimes agree with the corresponding numerical simulations of a twodimensional (2D) molecular system describing a finite-time Carnot cycle. From Eqs. (3) and (4), the total entropy change is given by

$$
\Delta S_{\mathrm{tot}}=-\frac{Q_{h}}{T_{h}}-\frac{Q_{c}}{T_{c}}=\frac{\Sigma_{h}}{t_{h}}+\frac{\Sigma_{c}}{t_{c}},
$$

whereas the efficiency is defined as usual:

$$
\eta=\frac{-W}{Q_{h}}=\frac{Q_{h}+Q_{c}}{Q_{h}} .
$$

In the present work we consider two different LDHE operation regimes: those that maximize power output $(P)[25,26]$ and the so-called $\Omega$ function [24] (which represents an optimal compromise between useful energy and lost energy), with

$$
\begin{gathered}
P=\frac{-W}{t_{c}+t_{h}}=\frac{Q_{h}+Q_{c}}{t_{h}+t_{c}}, \\
\Omega=\frac{(1+\tau) Q_{h}+2 Q_{c}}{t_{h}+t_{c}} .
\end{gathered}
$$


The contact times that optimize power output are [3]

$$
\begin{aligned}
& t_{h}^{\mathrm{MP}}=\frac{2 \Sigma_{h}}{\Delta S(1-\tau)}(1+\sqrt{\tau \Sigma}) \\
& t_{c}^{\mathrm{MP}}=\frac{2 \tau \Sigma \Sigma_{h}}{\Delta S(1-\tau)}\left(1+\sqrt{\frac{1}{\tau \Sigma}}\right)
\end{aligned}
$$

where $\Sigma \equiv \Sigma_{c} / \Sigma_{h}$ and $\tau=T_{c} / T_{h}$. Meanwhile, the contact times optimizing the $\Omega$ function are [24]

$$
\begin{aligned}
& t_{h}^{M \Omega}=\frac{2 \Sigma_{h}(1+\tau)}{\Delta S(1-\tau)}\left(1+\sqrt{\frac{2 \tau \Sigma}{1+\tau}}\right), \\
& t_{c}^{M \Omega}=\frac{4 \tau \Sigma \Sigma_{h}}{\Delta S(1-\tau)}\left(1+\sqrt{\frac{1+\tau}{\tau \Sigma}}\right) .
\end{aligned}
$$

After obtaining the optimal contact times, one can substitute into the expressions for $Q_{h}$ and $Q_{c}$, and into the efficiency definition, to get the efficiency in each performance regime. By doing so, it is straightforward to prove that

$$
\eta_{\mathrm{M} \Omega} \geqslant \eta_{\mathrm{MP}}
$$

and that the efficiency is respectively bounded as follows in the MP and $\mathrm{M} \Omega$ regimes:

$$
\begin{gathered}
\frac{\eta_{C}}{2} \leqslant \eta_{\mathrm{MP}} \leqslant \frac{\eta_{C}}{2-\eta_{C}}, \\
\frac{3 \eta_{C}}{4} \leqslant \eta_{\mathrm{M} \Omega} \leqslant \frac{3-2 \eta_{C}}{4-3 \eta_{C}} \eta_{C},
\end{gathered}
$$

where the lower bounds correspond to $\Sigma_{c} / \Sigma_{h} \rightarrow \infty$, while the upper bounds are obtained when $\Sigma_{c} / \Sigma_{h} \rightarrow 0$. Moreover, in the symmetric case $\left(\Sigma_{c}=\Sigma_{h}\right)$, the efficiencies of both regimes are $[3,24]$

$$
\begin{gathered}
\eta_{\mathrm{MP}}^{\mathrm{sym}}=1-\sqrt{1-\eta_{C}}=1-\sqrt{\tau}=\eta_{C A}, \\
\eta_{\mathrm{M} \Omega}^{\mathrm{sym}}=1-\sqrt{\frac{\left(1-\eta_{C}\right)\left(2-\eta_{C}\right)}{2}}=1-\sqrt{\frac{\tau(1+\tau)}{2}},
\end{gathered}
$$

where Eq. (14) was obtained in Ref. [2] for the maximum ecological regime.

\section{STABILITY BASED ON RESTITUTIVE FORCES THAT DEPEND ON THE HEAT EXCHANGED WITH THERMAL RESERVOIRS}

To perform the stability analysis of the LDHE model, in the two considered performance regimes, it is convenient to work with nondimensional thermodynamic variables. To this end let us rewrite the heat exchanged per cycle with thermal baths as

$$
\begin{gathered}
Q_{h}=T_{h} \Delta S\left(1-\frac{\Sigma_{h}}{\Delta S t_{h}}\right), \\
Q_{c}=-T_{h} \tau \Delta S\left(1+\frac{\Sigma_{h} \Sigma}{\Delta S t_{c}}\right) .
\end{gathered}
$$

Let us further define the following normalized (dimensionless) variables [29]:

$$
\begin{gathered}
\widetilde{t}_{c}=\frac{\Delta S}{\Sigma_{h}} t_{c}, \\
\widetilde{t}_{h}=\frac{\Delta S}{\Sigma_{h}} t_{h}, \\
\widetilde{Q}_{c}=\frac{Q_{c}}{T_{h} \Delta S}, \\
\widetilde{Q}_{h}=\frac{Q_{h}}{T_{h} \Delta S}, \\
\widetilde{\Delta S}_{\text {tot }}=\frac{\Delta S_{\text {tot }}}{\Delta S}, \\
\widetilde{\eta}=\eta, \\
\widetilde{P}=\frac{-\widetilde{Q}_{h}-\widetilde{Q}_{c}}{\widetilde{t}_{h}+\widetilde{t}_{c}}, \\
\widetilde{\Omega}=\frac{(1+\tau) \widetilde{Q}_{h}+2 \widetilde{Q}_{c}}{\widetilde{t}_{h}+\widetilde{t}_{c}} .
\end{gathered}
$$

With these definitions it is straightforward to get, after a little algebra, the following expressions for the new nondimensional variables:

$$
\begin{gathered}
\widetilde{Q}_{h}=\left(1-\frac{1}{\widetilde{t}_{h}}\right), \\
\widetilde{Q}_{c}=-\tau\left(1+\frac{\Sigma}{\widetilde{t}_{c}}\right), \\
\widetilde{\Delta S_{\text {tot }}}=\frac{1}{\widetilde{t}_{h}}+\frac{\Sigma}{\widetilde{t_{c}}}, \\
\tilde{\eta}=\frac{1-\tau-\frac{1}{t_{h}}-\frac{\tau \Sigma}{t_{c}}}{1-\frac{1}{\tau_{h}}}, \\
\widetilde{P}=\frac{1-\tau-\frac{1}{\tau_{h}}-\frac{\tau \Sigma}{\tau_{c}}}{t_{c}+t_{h}}, \\
\widetilde{\Omega}=\frac{1-\tau-\frac{1}{t_{h}}-\frac{2 \tau \Sigma}{\widetilde{t}_{c}}}{\widetilde{t}_{h}+\widetilde{t}_{c}} .
\end{gathered}
$$

Furthermore, substitution into Eqs. (8) and (9) respectively leads to the following expressions for the normalized contact times in the MP regime,

$$
\begin{gathered}
\widetilde{t}_{c}^{\mathrm{MP}}=\frac{2 \tau \Sigma}{1-\tau}\left(1+\frac{1}{\sqrt{\tau \Sigma}}\right), \\
\widetilde{t}_{h}^{\mathrm{MP}}=\frac{2}{1-\tau}(1+\sqrt{\tau \Sigma}),
\end{gathered}
$$

and those in the $\mathrm{M} \Omega$ regime,

$$
\begin{gathered}
\widetilde{t}_{c}^{M \Omega}=\frac{4 \tau \Sigma}{1-\tau}\left(1+\frac{1+\tau}{\sqrt{2 \tau \Sigma}}\right), \\
\widetilde{t}_{h}^{M \Omega}=\frac{2(1+\tau)}{1-\tau}\left(1+\sqrt{\frac{2 \tau \Sigma}{1+\tau}}\right) .
\end{gathered}
$$

Following Ref. [29] we assume that the control parameters of an LD engine model (i.e., the contact times with heat reservoirs) are dynamic variables governed via a dynamic system by design parameters like system size, conductivity of thermal 
resistors, heat capacity of thermal baths, and shape of potential energy landscapes. Although these control parameters can be in many cases handled with great precision under stability conditions, in some cases and especially at macroscopic scales, they could fluctuate due to external random perturbations the system is subject to. In these cases, we are interested in studying how these random fluctuations affect the system dynamic stability and thermodynamic performance under different operation regimes. Following the above discussion, we assume that the dynamics of $\widetilde{t_{c}}$ and $\widetilde{t_{h}}$ are governed by the amounts of heat exchanged in the corresponding isothermal process through a system of ordinary differential equations (ODEs) like the following:

$$
\begin{aligned}
& \frac{d \widetilde{t}_{c}}{d t}=f\left(\widetilde{Q}_{c}\left(\widetilde{t}_{c}, \widetilde{t}_{h}\right)\right), \\
& \frac{d \widetilde{t}_{h}}{d t}=g\left(\widetilde{Q}_{h}\left(\widetilde{t}_{c}, \widetilde{t}_{h}\right)\right) .
\end{aligned}
$$

Here and thereafter we use $*$ to indicate a steady-state value in any of the studied operation regimes, MP or M $\Omega$. In order to guarantee the stability of the stationary contact time values, $f$ and $g$ must be monotonically decreasing functions satisfying $f\left(\widetilde{Q}_{c}\left(\widetilde{t}_{c}^{*}, \widetilde{t}_{c}^{*}\right)\right)=g\left(\widetilde{Q}_{c}\left(\widetilde{t}_{h}^{*}, \widetilde{t}_{h}^{*}\right)\right)=0$. The simplest way to guarantee this is by assuming that the dynamics is described by the following linear (on $\widetilde{Q}_{c}$ and $\widetilde{Q}_{h}$ ) ODE system

$$
\begin{aligned}
\frac{d \widetilde{t}_{c}}{d t} & =A\left(\widetilde{Q}_{c}\left(\widetilde{t}_{c}^{*}, \widetilde{t}_{h}^{*}\right)-\widetilde{Q}_{c}\left(\widetilde{t}_{c}, \widetilde{t}_{h}\right)\right), \\
\frac{d \widetilde{t}_{h}}{d t} & =B\left(\widetilde{Q}_{h}\left(\tilde{t}_{c}^{*}, \widetilde{t}_{h}^{*}\right)-\widetilde{Q}_{h}\left(\widetilde{t}_{c}, \widetilde{t}_{h}\right)\right),
\end{aligned}
$$

where $A$ and $B$ are positive constants. These parameters determine the system response speed to perturbations from the steady state. Their values may depend on multiple characteristics, but usually the system size is the most important of them: the larger the system the smaller the values of $A$ and $B$. From a dynamical perspective, the inverse values of $A$ and $B$ set a characteristic time scale. As we will see in the forthcoming analyses, all of our results refer to this time scale, and so it is not necessary to specify $A$ and $B$ values. The local stability of the steady state of the dynamical system in Eqs. (37) and (38) is determined by the eigenvalues and eigenvectors of the corresponding Jacobian matrix [31-33,36]:

$$
\mathcal{J}=-\left[\begin{array}{ll}
\left.A \frac{\partial \widetilde{Q}_{c}}{\partial \tilde{t}_{c}}\right|_{\widetilde{t}_{c}^{*}, \widetilde{t}_{h}^{*}} & \left.A \frac{\partial \widetilde{Q}_{c}}{\partial \widetilde{t}_{h}}\right|_{\widetilde{t}_{c}^{*}, \widetilde{t}_{h}^{*}} \\
\left.B \frac{\partial \widetilde{Q}_{h}}{\partial \bar{t}_{c}}\right|_{\tilde{t}_{c}^{*}, \widetilde{t}_{h}^{*}} & \left.B \frac{\partial \widetilde{Q}_{h}}{\partial t_{h}}\right|_{\widetilde{t}_{c}^{*}, \widetilde{t}_{h}^{*}}
\end{array}\right] .
$$

After some algebra the resulting matrix for both operation regimes is

$$
\mathcal{J}=-\left[\begin{array}{cc}
-A \xi(\tau, \Sigma) & 0 \\
0 & -B \xi(\tau, \Sigma)
\end{array}\right]
$$

where

$$
\xi(\tau, \Sigma)= \begin{cases}\left(1+\frac{1-\tau}{2(\sqrt{\Sigma \tau})}\right)^{2}, & \text { MP } \\ \frac{1}{1+\tau}\left(\frac{1-\tau}{2}\left(1+\sqrt{\frac{2 \Sigma \tau}{1+\tau}}\right)\right)^{2}, & \mathrm{M} \Omega .\end{cases}
$$

Since $\mathcal{J}$ is diagonal, its eigenvalues are simply

$$
\lambda_{1}=-A \xi(\tau, \Sigma), \quad \lambda_{2}=-B \xi(\tau, \Sigma) .
$$

Observe that $\xi(\tau, \Sigma) \geqslant 0$, and so $\lambda_{1}$ and $\lambda_{2}$ are real and negative. This means that the system steady state is stable. Concomitantly, we can define the following relaxation times:

$$
t_{1,2} \equiv-\frac{1}{\lambda_{1,2}}
$$

The relaxation times for both operation regimes can be expressed in terms of $\eta_{C}=1-\tau$ and $\Sigma$ as

$$
t_{i}^{\mathrm{MP}}=\frac{4}{\eta_{C}^{2} I}\left(\sqrt{\Sigma\left(1-\eta_{C}\right)}+1\right)^{2}
$$

and

$$
t_{i}^{M \Omega}=\frac{4}{\eta_{C}^{2} I}\left(\frac{\sqrt{2 \Sigma\left(1-\eta_{C}\right)}}{2-\eta_{C}}+1\right)^{2}\left(2-\eta_{C}\right)
$$

where $i=\{1,2\}$ and $I=A$ if $i=1$, or $I=B$ if $i=2$.

The dependence of the above defined relaxation times (correspondingly normalized to either $A^{-1}$ or $B^{-1}$ ) on $\Sigma$ and $\eta_{C}$ is illustrated in Fig. 1(a). Notice that both relaxation times for the $\mathrm{M} \Omega$ regime are larger than those for the MP regime. This means that the steady state of an LDHE working at maximum power is more strongly stable than that of an engine operating at maximum $\Omega$, in the sense that the system returns faster to the steady state after any fluctuation. By contrasting this result with that in Eq. (10) we can see that modifying the engine operation regime to increase its efficiency affects its stability, because its dynamic response after a perturbation from the steady state is slowed down. The above discussion holds everywhere, except in the limits $\eta_{C} \rightarrow 0,1$ and $\Sigma \rightarrow 0, \infty$, where the relaxation times of both operation regimes achieve the same values. In these cases, from Eqs. (44) and (45),

$$
\begin{aligned}
\lim _{\eta_{C} \rightarrow 0} t_{i}^{*} & =\infty, \\
t_{i}^{*}\left(\eta_{C}=1\right) & =\frac{4}{I}, \\
\lim _{\Sigma \rightarrow \infty} t_{i}^{*} & =\infty, \\
t_{i}^{\mathrm{MP}}(\Sigma=0) & =\frac{4}{I \eta_{C}^{2}}, \\
t_{i}^{M \Omega}(\Sigma=0) & =\frac{4\left(2-\eta_{C}\right)}{I \eta_{C}^{2}} .
\end{aligned}
$$

From a dynamical point of view, a strong-stability criterion for LDHEs would be that, after a perturbation, the system returns to the steady state within a cycle period. By using Eqs. (31)-(34), we define the total times $\widetilde{t}_{\text {tot }}^{*} \equiv \widetilde{t}_{c}^{*}+\widetilde{t}_{h}^{*}$ for the $\mathrm{MP}$ and M $\Omega$ operation regimes, which result in

$$
\widetilde{t}_{\mathrm{tot}}^{*}= \begin{cases}\frac{2}{\eta_{C}}\left(1+\sqrt{\left(1-\eta_{C}\right) \Sigma}\right)^{2}, & \mathrm{MP}, \\ \frac{2\left(2-\eta_{C}\right)}{\eta_{C}}\left(1+\sqrt{\frac{\left(1-\eta_{C}\right) \Sigma}{2-\eta_{C}}}\right)^{2}, & \mathrm{M} \Omega .\end{cases}
$$

On the other hand, a total relaxation time, which we define as the summation of both relaxation times $t_{\text {relax }}^{*} \equiv t_{1}^{*}+t_{2}^{*}$ for the MP and M $\Omega$ regimes, can be computed by using Eqs. (44) 
(a)

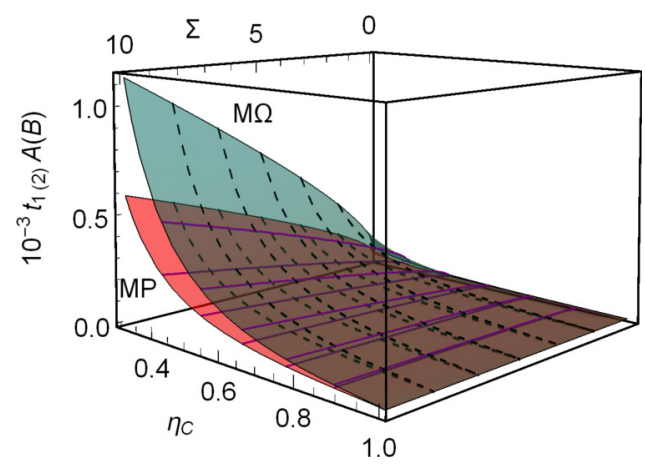

(b)

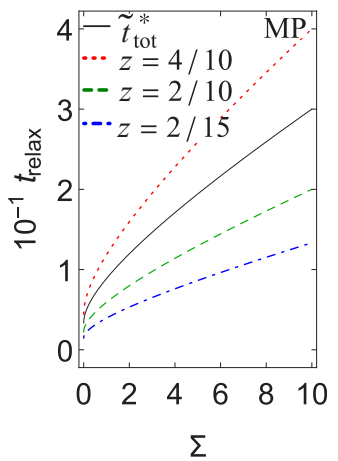

(c)

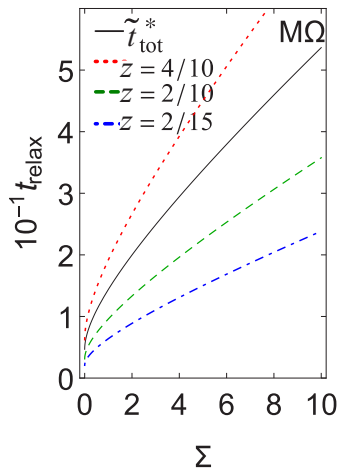

FIG. 1. (a) Plot of the relaxation times defined in Eq. (44) vs $\Sigma$ and $\eta_{C}$ for the MP and M $\Omega$ regimes. (b, c) Comparison between the stationary total cycle time, $\widetilde{t}^{*}$, and the total relaxation time corresponding to different values of $z=A^{-1}+B^{-1}$ for the (b) MP and (c) M $\Omega$ regimes. In (b) and (c) we took $\eta_{C}=3 / 5$. Total relaxation time is shorter than $\widetilde{t}^{*}$ whenever $z<\eta_{C} / 2=3 / 10$.

and (45). And after a little algebra we obtain that $t_{\text {relax }}^{*}$ is proportional to $\widetilde{t}_{\text {tot }}^{*}$ :

$$
t_{\text {relax }}^{*}=2\left(\frac{A^{-1}+B^{-1}}{\eta_{C}}\right) \widetilde{t}_{\mathrm{tot}}^{*}=\frac{2 z}{\eta_{C}} \widetilde{t}_{\mathrm{tot}}^{*},
$$

where $z \equiv A^{-1}+B^{-1}$. We wish to emphasize this last result, i.e., that the stability relaxation time and the cycle period are closely related, since as far as we are concerned it is one of the more relevant results of the present study. On the other hand, from the last two equations, the strong-stability condition, $\widetilde{t}_{\text {tot }}^{*}>t_{\text {relax }}^{*}$, becomes

$$
z<\frac{\eta_{C}}{2} \leqslant \frac{1}{2}
$$

This finding is illustrated in Figs. 1(b) and 1(c).

Summarizing, we have found that, in both the MP and the $\mathrm{M} \Omega$ regimes, there is a correlation between LDHE thermodynamic properties and the stability of its steady state. The system stability is enhanced (relaxation times are shortened) as $\Sigma \equiv \Sigma_{c} / \Sigma_{h}$ is decreased and/or $\eta_{C} \equiv$ $1-T_{c} / T_{h}$ is increased (see Fig. 1). Therefore, an optimal stability could be achieved when all of the entropy production is concentrated in the hot thermal bath and either the temperature of the hot bath tends to infinity or that of the cold bath tends to zero. On the other hand, the system efficiency is a growing function of $\eta_{C}$ and a decreasing function of $\Sigma$ (note that in the symmetric case, $\Sigma=1$, the irreversible dissipation at the hot bath produces less entropy than that occurring in the cold one-because the dissipated heat must be divided by temperature in order to get the entropy production). Thus, it seems that, regardless of the operation regime, manipulating the system design parameters has similar effects on the system stability strength and its efficiency.

From the dynamic equations [Eqs. (37) and (38)] one can see that the state $*$ is a fixed point, which is shown in Fig. 2(a) for MP and in Fig. 2(b) for M $\Omega$. After a perturbation to any state inside the physical region (where the combinations of $\alpha$ and $\widetilde{t}$ produce a valid power output $\widetilde{P}>0$ ), the system evolves towards the fixed point. Figure 2 shows that the stationary contact times with thermal baths are longer in the $\mathrm{M} \Omega$ than in the MP regime, which can be noticed when one compares the stationary states in Figs. 2(a) and 2(b) $\left(\widetilde{t}_{c, h}^{M \Omega}>\widetilde{t}_{c, h}^{\mathrm{MP}}\right)$. Thus, modifying the operation regime to increase the system efficiency (i.e., moving from maximum power to maximum $\Omega$ ) implies lengthening the cycle relaxation time. This suggests that changing from the operation regime to another with a de- (a)

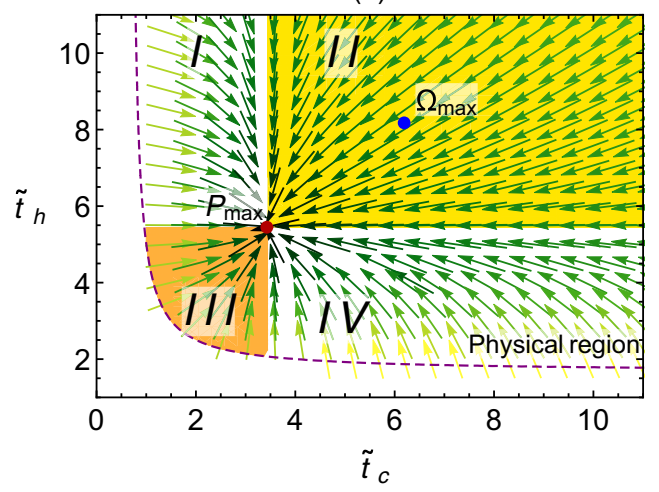

(b)

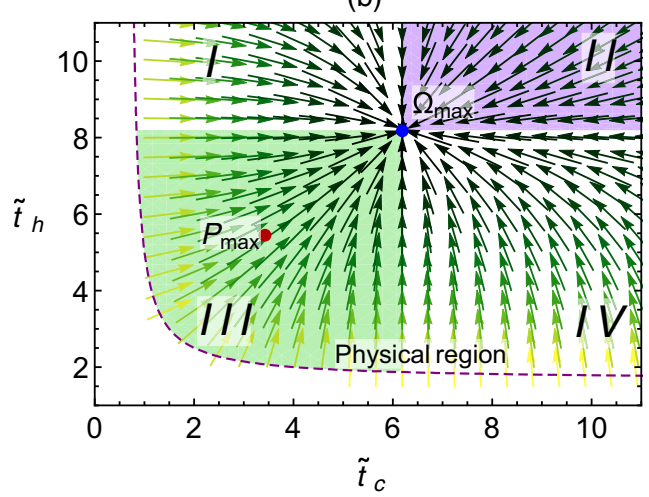

FIG. 2. Quiver plots of the velocity vector field given by the ODE system in Eqs. (37) and (38) with $\Sigma=1$ and $\tau=2 / 5$ for the (a) MP and (b) M $\Omega$ regimes. The MP and M $\Omega$ stationary states are explicitly indicated in both cases. Dashed lines denote the boundary of the useful-power-output region, $\widetilde{P}>0$. 
creased power output and increased efficiency slows down the engine dynamics altogether. Depending on the perturbation, the new location in the phase space of the system state point can be in any of the four regions depicted in the graphs of Fig. 2. Observe that the behavior of the relaxation trajectories after a perturbation is similar in the shaded and nonshaded regions, with a tendency in the shaded regions to slightly lean towards the line $\widetilde{t}_{c}=\widetilde{t}_{h}$ (i.e., the diagonal with slope 1 ), while in the nonshaded regions the trajectories slightly lean towards the quadrant where $\widetilde{t}_{c}>\widetilde{t}_{c}^{*}$ and $\widetilde{t}_{h}>\widetilde{t}_{h}^{*}$. In quadrant II of Fig. 2(a) (for MP), and Fig. 2(b) (for M $\Omega$ ) the relaxation occurs by increasing both contact times $\widetilde{t}_{c}$ and $\tilde{t}_{h}$; thus, the system total operation time always increases. In quadrant IV (for MP and M $\Omega$ ) the contact times decrease; thus, the total operation time decreases.

\section{STABILITY BASED ON THE HEAT FLUX INTO THE COLD RESERVOIR AND THE POWER OUTPUT}

In the present section we study the stability of an LDHE by considering that the system restitutive forces are functions of the heat flows between the engine and the thermal baths, rather than corresponding heat exchanges. To this end it is convenient to consider the total cycle time and the contact time with the cold reservoir as dynamic variables whose time derivatives are functions of the engine power output and heat flux to the cold temperature bath [29]. Let us define the following dimensionless variables:

$$
\widetilde{\Sigma}_{c} \equiv \Sigma_{c} / \Sigma_{T}, \quad \alpha \equiv t_{c} /\left(t_{c}+t_{h}\right), \quad \tilde{t} \equiv \frac{\Delta S}{\Sigma_{T}}\left(t_{c}+t_{h}\right),
$$

with $\Sigma_{T} \equiv \Sigma_{h}+\Sigma_{c}$. This allows us to write the following expressions for dimensionless thermodynamic variables:

$$
\begin{gathered}
\dot{\widetilde{Q}}_{h} \equiv \frac{Q_{h}}{\widetilde{t} T_{c} \Delta S}=\left(1-\frac{1-\widetilde{\Sigma}_{c}}{(1-\alpha) \widetilde{t}}\right) \frac{1}{\tau \widetilde{t}}, \\
\dot{\widetilde{Q}}_{c} \equiv \frac{Q_{c}}{\widetilde{t} T_{c} \Delta S}=-\left(1+\frac{\widetilde{\Sigma}_{c}}{\alpha \widetilde{t}}\right) \frac{1}{\widetilde{t}}, \\
\dot{\widetilde{\Delta} S_{\text {tot }}}=\frac{1}{\widetilde{t}^{2}}\left(\frac{1-\widetilde{\Sigma}_{c}}{1-\alpha}+\frac{\widetilde{\Sigma}_{c}}{\alpha}\right), \\
\widetilde{\eta}=\eta=1-\tau \frac{1+\frac{\widetilde{\Sigma}_{c}}{\alpha \widetilde{t}}}{1-\frac{1-\widetilde{\Sigma}_{c}}{(1-\alpha) \widetilde{t}}} \\
\widetilde{P}=\frac{1}{\tau \widetilde{t}}\left(1-\tau-\frac{1-\widetilde{\Sigma}_{c}}{(1-\alpha) \widetilde{t}}-\frac{\tau \widetilde{\Sigma_{c}}}{\alpha \widetilde{t}}\right), \\
\widetilde{\Omega}=\frac{1-\tau}{\tau \widetilde{t}}-\frac{(1+\tau)\left(1-\widetilde{\Sigma}_{c}\right)}{(1-\alpha) \widetilde{t}^{2}}-\frac{2 \widetilde{\Sigma}_{c}}{\alpha \widetilde{t}^{2}} .
\end{gathered}
$$
$\widetilde{\Omega}$ are

In this scheme, the values of $\alpha$ and $\tilde{t}$ that maximize $\widetilde{P}$ and

$$
\begin{gathered}
\alpha^{*}= \begin{cases}\frac{1}{1+\sqrt{\frac{1-\widetilde{\Sigma}_{c}}{\tau \Sigma_{c}}},} & \mathrm{MP}, \\
\frac{1}{1+\sqrt{\frac{(1+\tau)\left(1-\widetilde{\Sigma}_{c}\right)}{2 \tau \Sigma_{c}}}}, & \mathrm{M} \Omega,\end{cases} \\
\widetilde{t}^{*}= \begin{cases}\frac{2}{1-\tau}\left(\sqrt{\tau \widetilde{\Sigma}_{c}}+\sqrt{1-\widetilde{\Sigma}_{c}}\right)^{2}, & \mathrm{MP}, \\
\frac{2}{1-\tau}\left(\sqrt{\left.2 \tau \widetilde{\Sigma}_{c}+\sqrt{\left(1-\widetilde{\Sigma}_{c}\right)(1+\tau)}\right)^{2},},\right. & \mathrm{M} \Omega .\end{cases}
\end{gathered}
$$

Following the derivation in the previous section we assume that the restitutive forces for dynamic variables $\alpha$ and $\widetilde{t}$ are linear functions of $\dot{\widetilde{Q}}_{c}$ and $\widetilde{P}$ :

$$
\begin{aligned}
& \frac{d \alpha}{d t}=C\left(\dot{\widetilde{Q}}_{c}\left(\alpha^{*}, \widetilde{t}^{*}\right)-\dot{\widetilde{Q}}_{c}(\alpha, \widetilde{t})\right), \\
& \frac{d \widetilde{t}}{d t}=D\left(\widetilde{P}\left(\alpha^{*}, \widetilde{t}^{*}\right)-\widetilde{P}(\alpha, \widetilde{t}) x\right),
\end{aligned}
$$

where * indicates the corresponding steady-state values, while $C$ and $D$ are positive constants. The meaning of these parameters is similar to that of $A$ and $B$ in the former section. From previous works we know that constraints on $\alpha$ are linked to endoreversible behaviors of the heat engine (open parabolic $P$ vs $\eta$ curves). Then, perturbations of this variable are expected to reproduce a stable response, just like in endoreversible engines reported in the literature [37]. On the other hand, constraints on the operation time $\tilde{t}$ are likened to the irreversible character of the engine. Thus, perturbations of this other variable are related to spontaneous changes in the irreversibility of the system, which might not lead to stable points for large perturbations, as shown below.

As in the previous case, in a linear approximation, the local stability of the steady state is determined by the Jacobian matrix

$$
\mathcal{J}=-\left[\begin{array}{cc}
\left.C \frac{\partial \dot{\widetilde{Q}}_{c}}{\partial \alpha}\right|_{\alpha^{*}, \widetilde{t}^{*}} & \left.C \frac{\partial \dot{\widetilde{Q}}_{c}}{\partial t}\right|_{\alpha^{*}, \tilde{t}^{*}} \\
\left.D \frac{\partial \widetilde{P}}{\partial \alpha}\right|_{\alpha^{*}, \tilde{t}^{*}} & \left.D \frac{\partial \widetilde{P}}{\partial \vec{t}}\right|_{\alpha^{*}, \tilde{t}^{*}}
\end{array}\right] .
$$

In the MP case, $\mathcal{J}$ has a null row. This happens because, by definition, in the MP regime $\left.\frac{\partial \widetilde{P}}{\partial \alpha}\right|_{P_{\max }}=\left.\frac{\partial \widetilde{P}}{\partial \widetilde{t}}\right|_{P_{\max }}=0$. Thus, the linearized dynamical system becomes

$$
\begin{gathered}
\frac{d \alpha}{d t}=\left.C \frac{\partial \dot{\tilde{Q}_{c}}}{\partial \alpha}\right|_{P_{\max }} \alpha+\left.C \frac{\partial \dot{\widetilde{Q}}_{c}}{\partial \widetilde{t}}\right|_{P_{\max }} \tilde{t}, \\
\frac{d \tilde{t}}{d t}=0,
\end{gathered}
$$

resulting in $\tilde{t}$ preserving its initial condition value. Conversely, in the $\mathrm{M} \Omega$ regime, the Jacobian second row is not zero:

$$
\begin{aligned}
\frac{d \alpha}{d t} & =\left.C \frac{\partial \dot{\widetilde{Q}}_{c}}{\partial \alpha}\right|_{\Omega_{\max }} \alpha+\left.C \frac{\partial \dot{\widetilde{Q}}_{c}}{\partial \widetilde{t}}\right|_{\Omega_{\max }} \tilde{t}, \\
\frac{d \widetilde{t}}{d t} & =\left.D \frac{\partial \widetilde{P}}{\partial \alpha}\right|_{\Omega_{\max }} \alpha+\left.D \frac{\partial \widetilde{P}}{\partial \widetilde{t}}\right|_{\Omega_{\max }} \tilde{t}
\end{aligned}
$$

However, after some algebra one obtains that the Jacobian rows are linearly dependent, and so

$$
\mathcal{J}^{M \Omega}=\Gamma\left[\begin{array}{cc}
-1 & -\nu \\
\frac{\gamma(1-\tau)}{(1+\tau)} & \frac{\gamma(1-\tau)}{(1+\tau)} v
\end{array}\right],
$$

where

$$
\begin{aligned}
& \gamma \equiv D / C, \quad \Gamma \equiv-\frac{C(1-\tau)^{2}}{8 \tau\left(\sqrt{2 \tau \widetilde{\Sigma}_{\mathrm{c}}}+\sqrt{\left(1-\widetilde{\Sigma}_{\mathrm{c}}\right)(1+\tau)}\right)^{2}} \\
& \nu \equiv \frac{(1+\tau) \sqrt{2 \tau \widetilde{\Sigma}_{\mathrm{c}}}+2 \tau \sqrt{\left(1-\widetilde{\Sigma}_{\mathrm{c}}\right)(1+\tau)}}{\left(\sqrt{2 \tau \widetilde{\Sigma}_{\mathrm{c}}}+\sqrt{\left(1-\widetilde{\Sigma}_{\mathrm{c}}\right)(1+\tau)}\right)^{3}}
\end{aligned}
$$




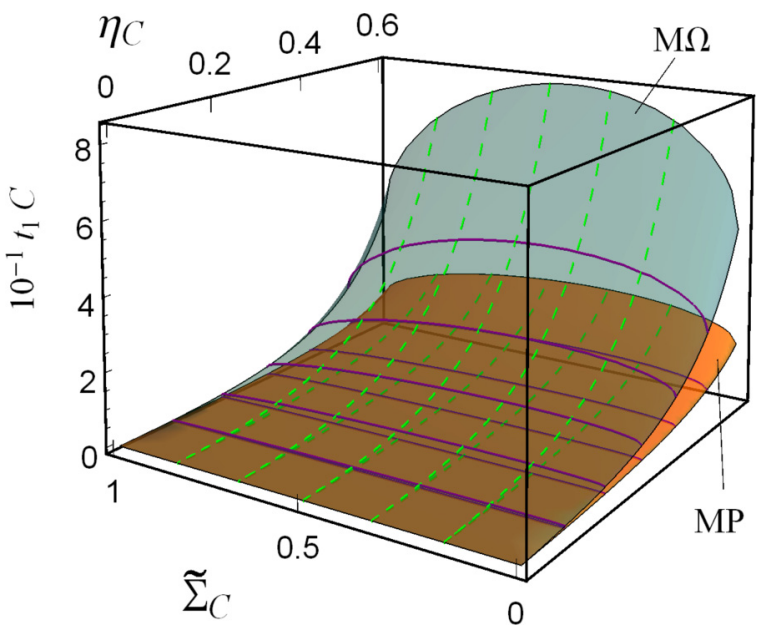

FIG. 3. Plot of the relaxation times defined in Eq. (71) vs $\widetilde{\Sigma}_{c}$ and $\eta_{C}$ for the MP and $\mathrm{M} \Omega$ regimes.

One can straightforwardly verify that the determinant of $\mathcal{J}$ is zero for the two considered operation regimes. This in turn means that one of the $\mathcal{J}$ eigenvalues equals zero. On the other hand, the nonzero eigenvalue of the Jacobian matrix, for each of the operation regimes, is given by

$$
\lambda= \begin{cases}\left.C \frac{\partial \dot{\widetilde{Q}}_{c}}{\partial \alpha}\right|_{\alpha^{\mathrm{MP}}, \bar{t}^{\mathrm{MP}}}, & \mathrm{MP}, \\ \left.C^{\prime} \frac{\partial \widetilde{\mathscr{Q}}_{c}}{\partial \alpha}\right|_{\alpha^{M \Omega}, \tilde{t}^{M \Omega}}, & \mathrm{M} \Omega .\end{cases}
$$

The fact that one of the Jacobian eigenvalues is zero implies that the system trajectories move with zero speed along the corresponding eigendirection. Therefore, trajectories starting in the neighborhood of the steady state would move parallel to the eigendirection with nonzero eigenvalue, until they intersect the eigendirection with null eigenvalue and stop there. In other words, the system steady state is not isolated, but surrounded by an infinite number of steady states that are aligned along the zero-eigenvalue eigendirection. A relaxation time for the approach of phase-space trajectories to the nulleigenvalue eigendirection can be defined as $t_{1}=-1 / \lambda$. After a little algebra, the value this relaxation time achieves for each of the studied operation regimes is

$$
t_{1}= \begin{cases}\frac{2\left(1-\eta_{C}\right)}{C \eta_{C}} \tilde{t}^{\mathrm{MP}}, & \mathrm{MP}, \\ \frac{4\left(1-\eta_{C}\right)}{C^{\prime} \eta_{C}} \tilde{t}^{M \Omega}, & \mathrm{M} \Omega .\end{cases}
$$

Although with a different meaning, a strong-stability constraint can be imposed as follows: $t_{1}^{*} \leqslant \widetilde{t}^{*}$. As we have discussed, the steady state is not isolated in this case. Thus, (a)

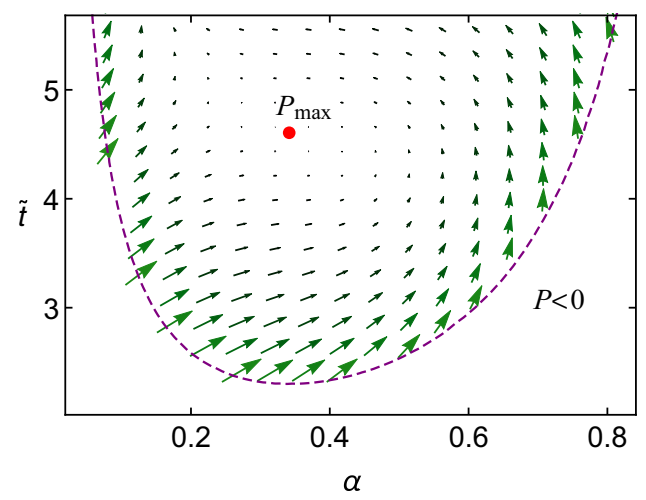

(c)

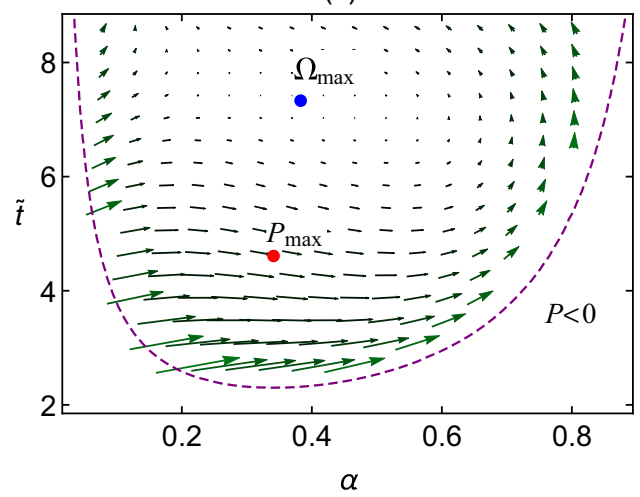

(b)

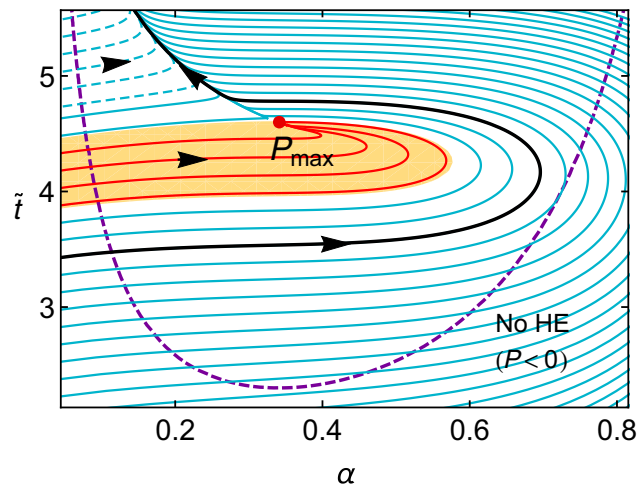

(d)

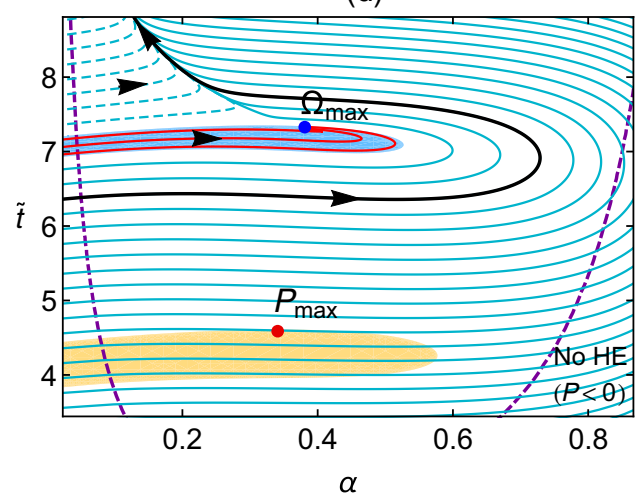

FIG. 4. Left: Quiver plots of the velocity vector field of the dynamical system given in Eqs. (62) and (63) correspond to (a) the MP regime and (c) the M $\Omega$ regime. Right: Plots of numerically computed phase-space trajectories corresponding to (b) the MP regime and (d) the M $\Omega$ regime. For all these plots we use $C=D=1$, and $\widetilde{\Sigma}_{c}=\tau=2 / 5$. In (b) and (d) the upper (blue) and lower (orange) shaded areas indicate the attraction basins of the null-eigenvalue eigendirection for the MP and M $\Omega$ regimes, respectively. Dashed lines denote the boundary of the useful-power-output region, $\widetilde{P}>0$. 

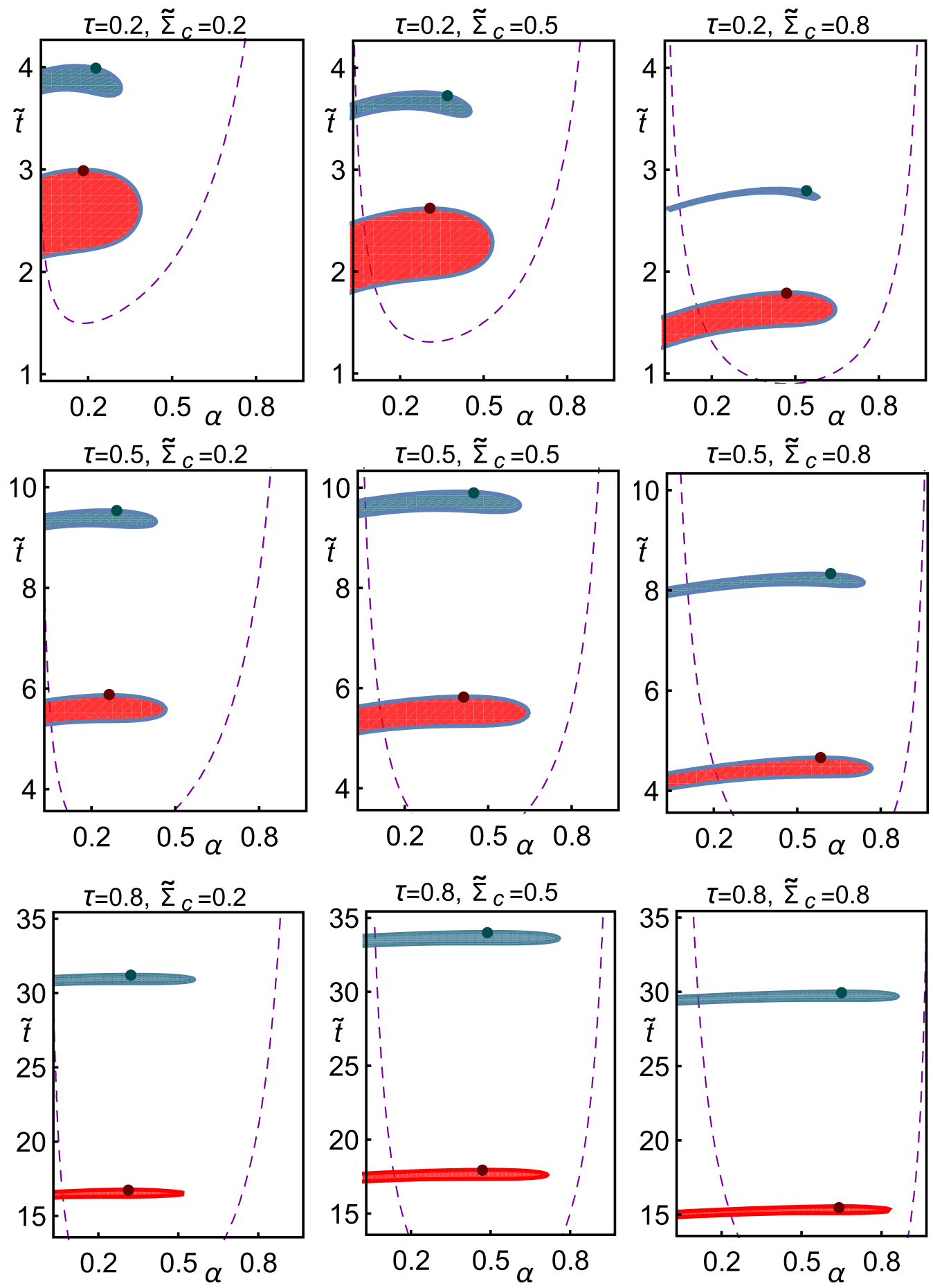

FIG. 5. Evolution of the basin of attraction for the MP and M $\Omega$ cases [lower (red) and upper (blue) basins of stability, respectively] using some $\tau$ and $\widetilde{\Sigma}_{\mathrm{c}}$ values.

the strong-stability constraint indicates that, after a perturbation, the point denoting the system state in the phase space would return to the null-eigenvalue eigendirection within the same cycle. From Eqs. (71), the strong-stability constraint requires constants $C$ and $C^{\prime}$ (corresponding to the MP and $\mathrm{M} \Omega$ regimes) to satisfy the following inequalities:

$$
C>\frac{2\left(1-\eta_{C}\right)}{\eta_{C}}, \quad C^{\prime}>\frac{4\left(1-\eta_{C}\right)}{\eta_{C}} .
$$

In Fig. 3, we show plots of $t_{1}$ (correspondingly normalized to either $C^{-1}$ or $C^{\prime-1}$ ) vs $\widetilde{\Sigma}_{c}$ and $\eta_{C}$. Notice that, in general, relaxation times in the $\mathrm{M} \Omega$ regime are one order of magnitude larger than those of the MP regime. This agrees with our previous observation that departing from the maximum-power regime to decrease entropy production and increase thermodynamic efficiency implies a cost in terms of stability strength. To our consideration, the observed increase of stability as the engine power output (efficiency) increases (decreases) is due to larger heat flows through the system, which in 
(a)

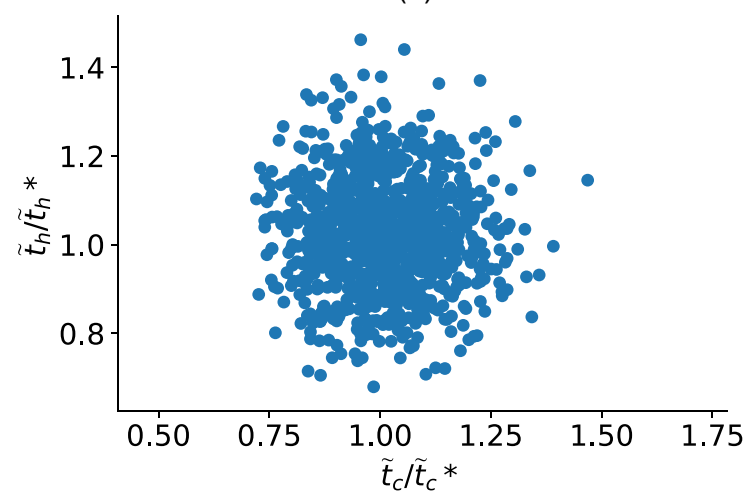

(c)

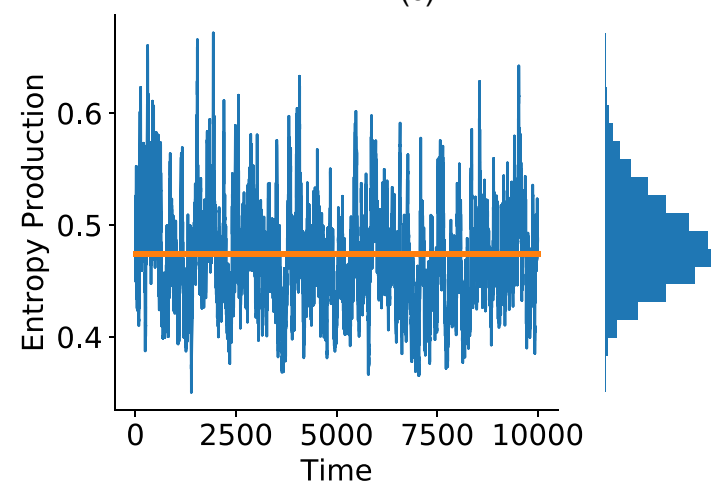

(b)

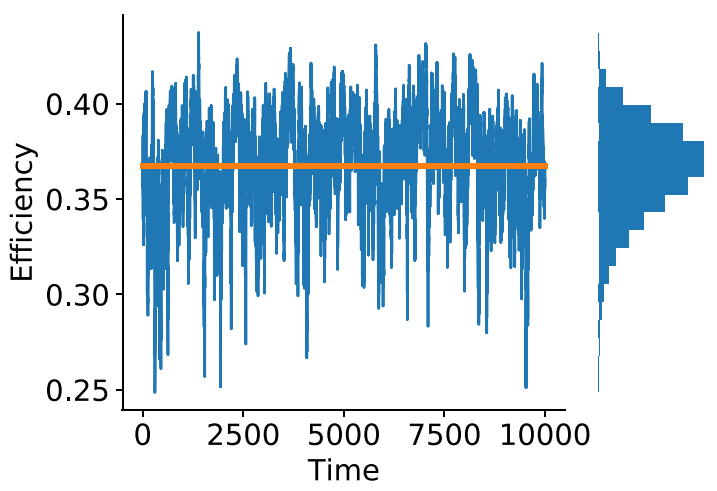

(d)

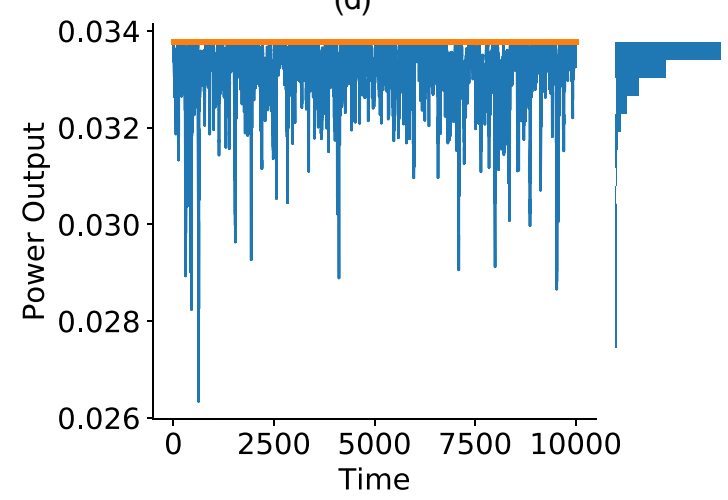

FIG. 6. Results of numerical simulations of an LDHE working in the MP regime with additive white noise. In this case, restitutive forces are dependent on the heat exchanged between the engine and the thermal baths. (a) Scatterplot of $\tilde{t}_{h} / \tilde{t}_{h}^{\mathrm{MP}}$ vs $\tilde{t}_{c} / \tilde{t}_{c}^{\mathrm{MP}}$. Plots of (b) efficiency, (c) entropy production, and (d) power output, together with the corresponding probability distribution functions. Horizontal lines (orange) denote stationary-state values.

turn imply larger values of the right-hand sides in the ODEs governing the system dynamics. Also notice that, in both the MP and the M $\Omega$ regimes, the relaxation time achieves its maximum at a value of $\widetilde{\Sigma}_{c}$ intermediate between zero and 1. Therefore, one way to strengthen the system stability is to increase the asymmetry on entropy production in the thermal baths. In this way, stability considerations can be included in the control parameter analysis of the MP and M $\Omega$ operation regimes. Interestingly, an increase of asymmetry has been related to larger (smaller) power output with smaller (larger) efficiencies, as can be seen in Fig. 2 of Ref. [26]. Observe as well that the relaxation time is a monotonic decreasing function of Carnot's efficiency in both regimes of operation. Therefore, by manipulating the engine thermal baths so that the value of $\eta_{C}$ increases, one can strengthen the system dynamic stability. Furthermore, the above discussion suggests that an optimal ratio for the thermal bath temperatures should exist to optimize the system stability. As we have seen, smaller temperature ratios take the system closer to the reversible limit and improve its stability, but getting too close to the reversible limit highly increases the system operation time, and this in turn has deleterious effects because it slows down the system stability; recall that the relaxation times scale with the engine operation time [Eqs. (71)].

The discussion in the previous paragraphs is based on a local stability analysis of the LDHE fixed point, and therefore it is only valid for initial conditions in the neighborhood of the steady state. To expand our understanding of the system dynamics we numerically explored the system behavior by taking into consideration numerous different initial conditions. The dynamics resulting from the ODE system given by Eqs. (62) and (63) is depicted in Fig. 4 for the MP and M $\Omega$ performance regimes. Observe that in this case the system behavior greatly differs from the case in which restitutive forces depend on the amount of heat exchanged per cycle between the thermal engine and the heat baths (compare Figs. 2 and 4). In the first case the system steady state is globally stable, whereas we can appreciate in Fig. 4 a more complex behavior. There exists a basin of attraction such that all trajectories starting within it converge to a neighborhood of the steady state, while trajectories starting outside the basin of attraction rapidly converge to a one-dimensional manifold in the phase space (that corresponds to the $d \alpha / d t$ nullcline) and slowly diverge to $\alpha \rightarrow 0$ and $\tilde{t} \rightarrow \infty$ along this manifold. This last observation further implies that the contact time with the cold (hot) temperature bath slowly decreases (increases) until the cycling period diverges to infinity and the engine reaches an adiabatic regime. The above described behavior occurs in the two studied regimes of operation. Since the steady states of the MP and M $\Omega$ regimes are different, it is natural that the location of the corresponding basin of attraction changes. However, to our understanding, it is not so obvious why the basin of attraction in the $\mathrm{M} \Omega$ regime is notably narrower than that of the MP regime. We believe this has to do with the 


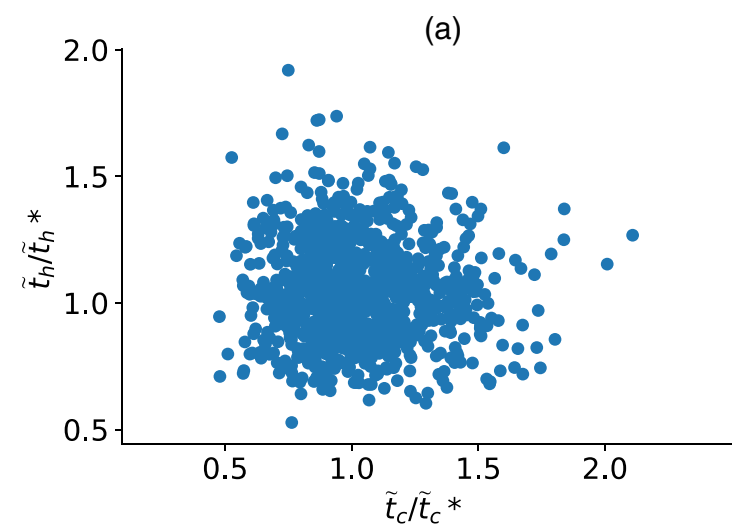

(c)

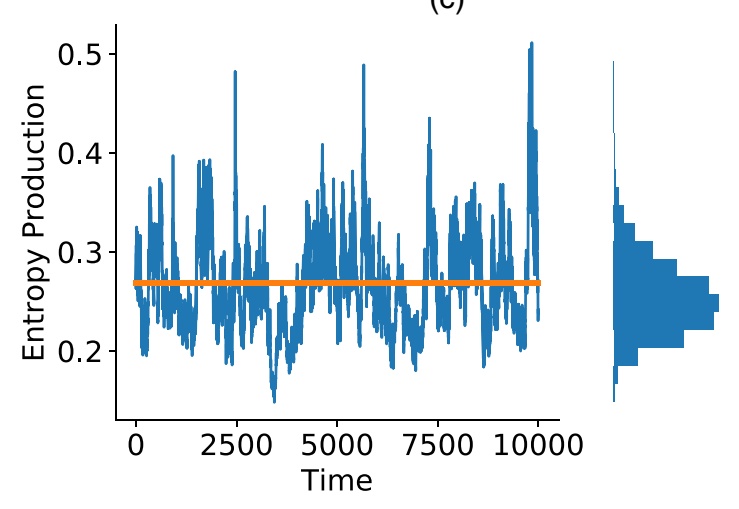

(b)

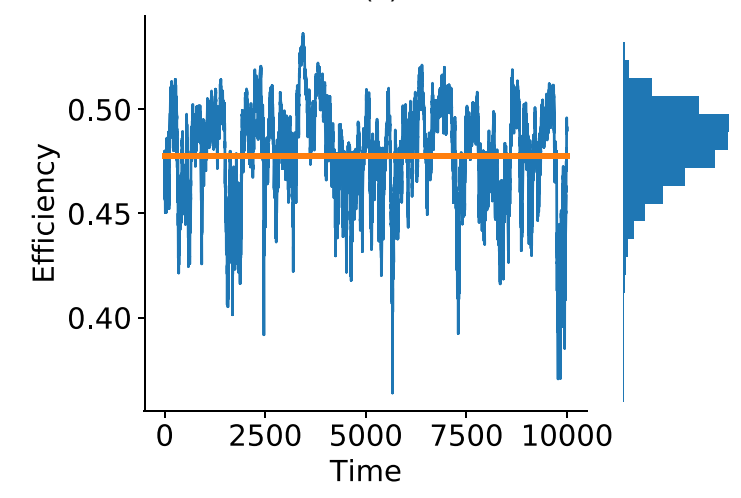

(d)

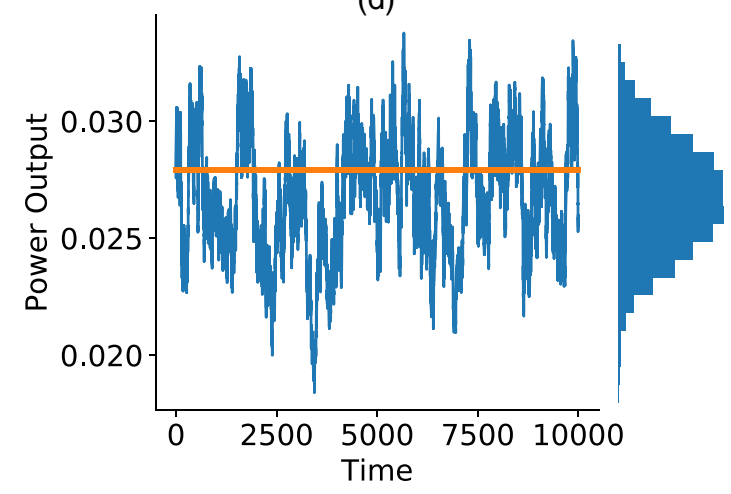

FIG. 7. Results of numerical simulations of an LDHE working in the M $\Omega$ regime with additive white noise. In this case, restitutive forces are dependent on the heat exchanged between the engine and the thermal baths. (a) Scatterplot of $\tilde{t}_{h} / \tilde{t}_{h}^{\mathrm{MP}}$ vs $\tilde{t}_{c} / \tilde{t}_{c}^{\mathrm{MP}}$. Plots of (b) efficiency, (c) entropy production, and (d) power output, together with the corresponding probability distribution functions. Horizontal lines (orange) denote stationary-state values.

fact that $\eta_{\mathrm{M} \Omega} \geqslant \eta_{\mathrm{MP}}$ [see Eq. (10)]. As the engine efficiency approaches $\eta_{C}$, more restrictions are expected to constraint the system, and one could expect the basin of attraction to shrink in consequence. A detailed analysis of the evolution of the basin of attraction as the values of $\widetilde{\Sigma}_{c}$ and $\tau$ change is shown in Fig. 5. Observe how in every graph of this figure the basin of attraction is narrower for the M $\Omega$ than for the MP regime. Observe as well that for increasing $\tau$ (decreasing $\eta_{C}$ ) the difference between the basins of attraction of both regimes gets smaller. Furthermore, as $\Sigma_{c}$ increases, both basins of attraction increase their length in the $\alpha$ direction. Finally, the basins of attraction for both regimes become narrower as $\tau$ decreases $\left(\eta_{C}\right.$ increases), which is not obvious in the figure due to the change of scale in the $\tilde{t}$ axis.

The comparison of the basin of attractions for different values of $\tau$ and $\widetilde{\Sigma}_{c}$ is presented in Fig. 5 where the cases $\tau=\{0.2,0.5,0.8\}$ and $\Sigma_{\mathrm{c}}=\{0.2,0.5,0.8\}$ are depicted.

\section{DYNAMICS OF AN LDHE SUBJECT TO RANDOM PERTURBATIONS}

In the previous sections we have studied how LDHE dynamic stability and thermodynamic properties are affected by the system design parameters. We did this considering two different operation regimes and two different restitutive-force scenarios. The analyzed operation regimes are maximum power and maximum $\Omega$ function — this last regime represents an optimal compromise between high power output and low entropy production. On the other hand, the scenarios for the restitutive forces are that they (1) depend on the amount of heat exchanged per cycle between the engine and the thermal baths and (2) depend on heat fluxes between the engine and the thermal baths. These analyses allowed us to understand the system dynamic response after a perturbation from the steady state. However, it is also important to analyze the behavior of the system thermodynamic variables when the system dynamic state is subject to random perturbations. Taking this into consideration, we simulated the dynamics of an LDHE in all four combinations of operation regimes and restitutiveforce scenarios, by numerically solving the corresponding dynamical system and taking into account random perturbations in the form of additive white noise. To do this we employed Euler's algorithm and added a normally distributed random number to every variable in each algorithm iteration. More specifically, consider the dynamical system

$$
\begin{aligned}
& \dot{x}=f(x, y), \\
& \dot{y}=g(x, y) .
\end{aligned}
$$

Then, by approximating the time derivatives as difference ratios the above system can be rewritten as

$$
x_{i+1}=x_{i}+f\left(x_{i}, y_{i}\right) \Delta t, \quad y_{i+1}=y_{i}+g\left(x_{i}, y_{i}\right) \Delta t
$$


(a)

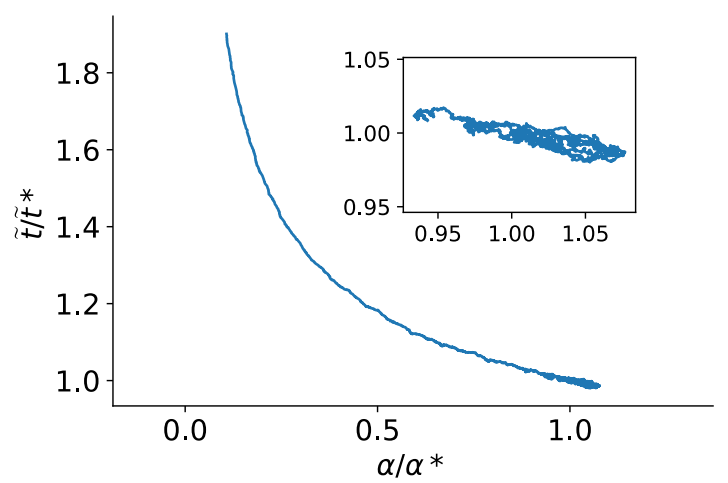

(c)

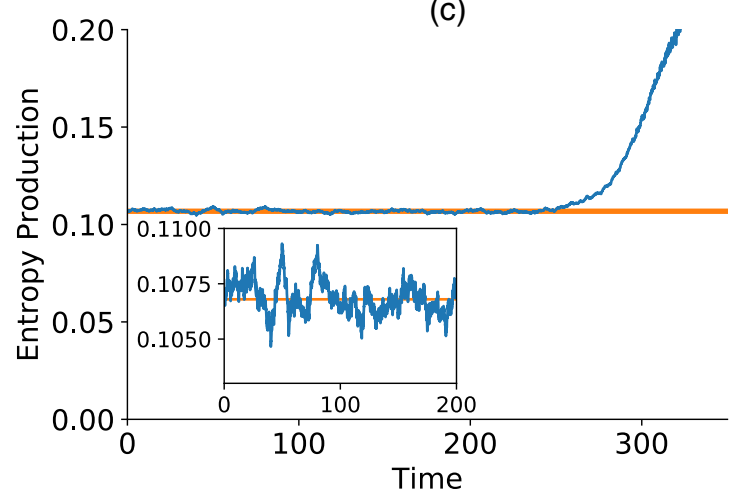

(b)

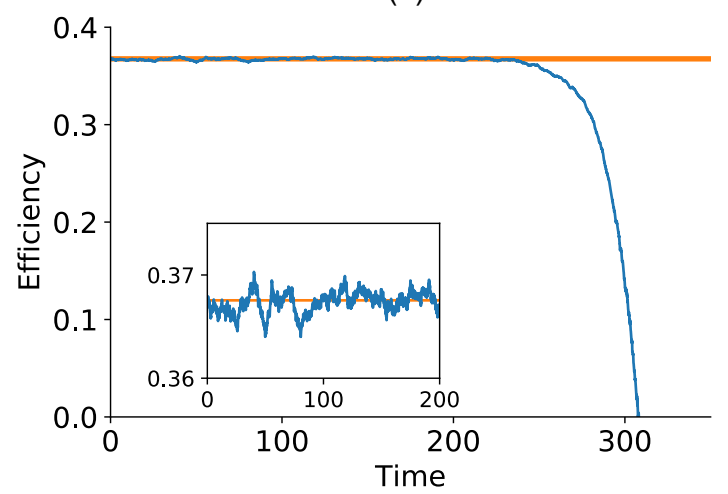

(d)

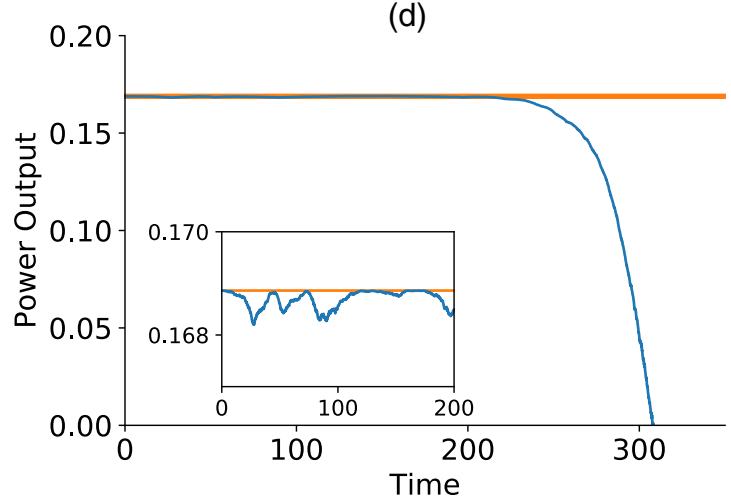

FIG. 8. Results of numerical simulations of an LDHE working in the MP regime with additive white noise. In this case, restitutive forces are dependent on the values of heat flux into the cold reservoir and power output. (a) System trajectory in the $\left(\tilde{t} / \tilde{t}^{\mathrm{MP}}, \alpha / \alpha^{\mathrm{MP}}\right)$ space. Plots of (b) efficiency, (c) entropy production, and (d) power output. Horizontal lines (orange) denote stationary-state values.

From this, one can include random perturbations in the form of additive white noise as follows according to the wellknown Euler-Maruyama method,

$$
\begin{aligned}
& x_{i+1}=x_{i}+f\left(x_{i}, y_{i}\right) \Delta t+\xi \sqrt{\Delta t}, \\
& y_{i+1}=y_{i}+g\left(x_{i}, y_{i}\right) \Delta t+\zeta \sqrt{\Delta t},
\end{aligned}
$$

where $\xi$ and $\zeta$ are normally distributed random variables with zero mean values and standard deviations as follows:

$$
\sigma_{\xi}=c_{v} x^{*} \quad \text { and } \quad \sigma_{\zeta}=c_{v} y^{*} .
$$

As before, $*$ denotes the steady-state value of the corresponding variable, while $c_{v}$ is a coefficient of variation that measures the additive noise intensity. From the above discussion, the Euler-Maruyama algorithm to solve the system of differential equations with the inclusion of additive normally distributed random noise is as follows:

(i) Choose the variable initial values, $x_{0}$ and $y_{0}$, and the time step interval $\Delta t$.

(ii) For a given $i=0,1,2 \ldots$, compute

$$
\begin{aligned}
& x_{i+1}=x_{i}+f\left(x_{i}, y_{i}\right) \Delta t+\xi \sqrt{\Delta t}, \\
& y_{i+1}=y_{i}+g\left(x_{i}, y_{i}\right) \Delta t+\zeta \sqrt{\Delta t} .
\end{aligned}
$$

(iii) Iterate (ii).

Below we discuss the results of our numerical simulations.

\section{A. Restitutive forces dependent on the heat exchanged between the engine and the thermal baths}

By employing the above described algorithm we numerically solved the differential equation system (37) and (38), with $\widetilde{t}_{c}^{*}$ and $\widetilde{t}_{h}^{*}$ as given by Eqs. (31) and (32) for the MP regime, and by Eqs. (33) and (34) for the $\mathrm{M} \Omega$ regime. In all cases we took $\tau=2 / 5, \Sigma=1, c_{v}=0.03$, and $\Delta t=0.1$, and the initial condition was set to the steady state. After solving the differential equations for contact times $\widetilde{t}_{c}$ and $\widetilde{t}_{h}$, we substituted into Eqs. (27)-(29) to obtain expressions

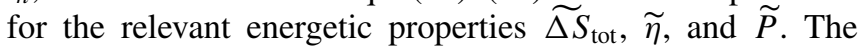
obtained results for the MP regime are shown in Fig. 6. Observe that the phase-space point representing the system state moves on a more-or-less circular surface around the steady state due to the additive white noise. Furthermore, the efficiency fluctuates around the corresponding steady-state value, with a slightly larger probability to fluctuate towards smaller values. Conversely, the entropy production per cycle shows a slightly larger tendency to fluctuate towards values larger than the steady-state value. Finally, the power output decreases in every fluctuation. This last result immediately follows from the fact that the steady state corresponds to the maximum-power regime.

We also solved the system differential equations for the $\mathrm{M} \Omega$ regime, using the same parameter values and the same initial condition as in the case of the MP regime. The results are shown in Fig. 7. We can appreciate there that these results are qualitatively equivalent to those of Fig. 6. However, 
(a)

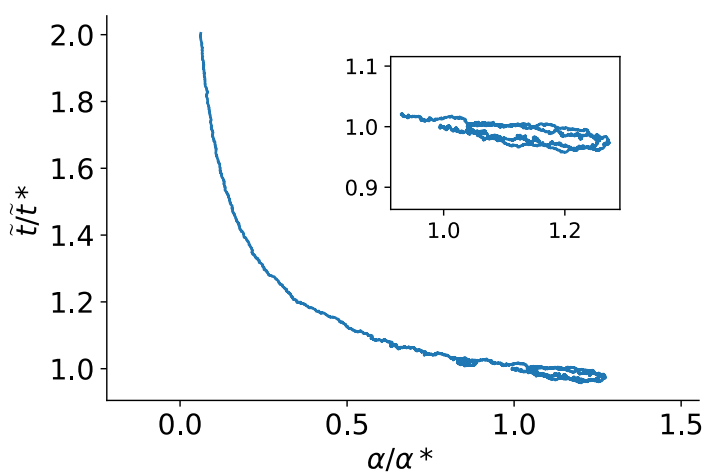

(c)

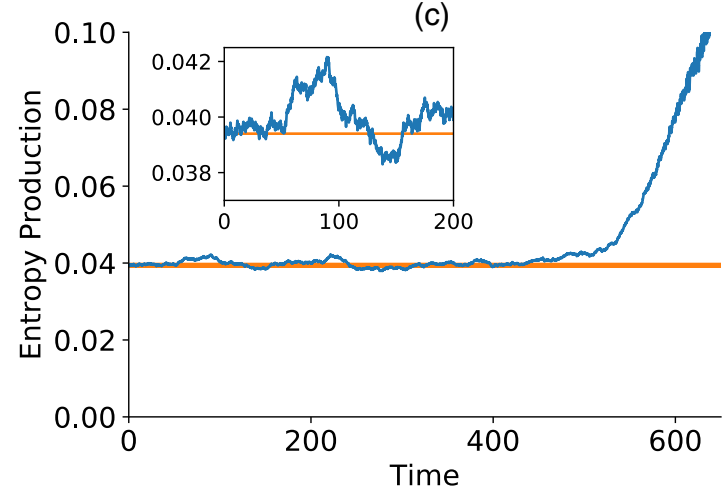

(b)

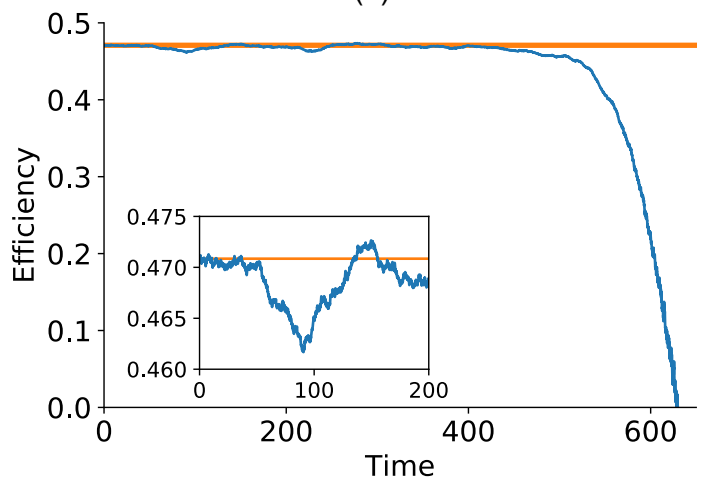

(d)

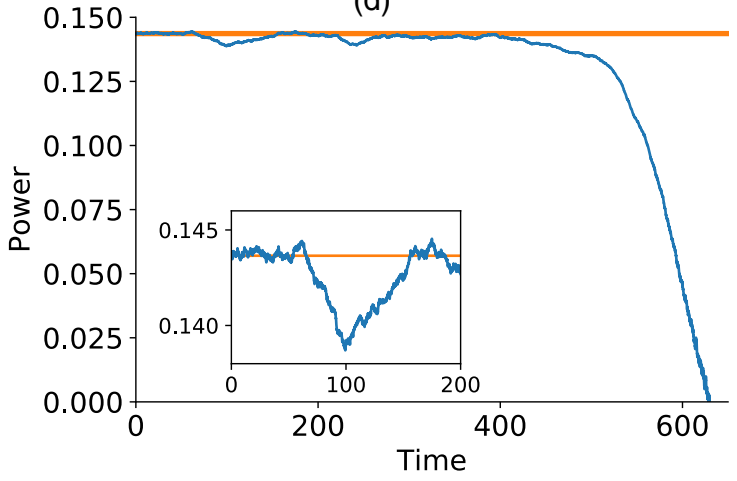

FIG. 9. Results of numerical simulations of an LDHE working in the M $\Omega$ regime with additive white noise. In this case, restitutive forces are dependent on the values of heat flux into the cold reservoir and power output. (a) System trajectory in the $\left(\widetilde{t} / \widetilde{t}^{\mathrm{MP}}, \alpha / \alpha^{\mathrm{MP}}\right)$ space. Plots of (b) efficiency, (c) entropy production, and (d) power output. Horizontal lines (orange) denote stationary-state values.

some interesting observations result from a closer comparison between Figs. 6 and 7:

(a) The $\mathrm{M} \Omega$ regime of operation is more efficient, is less powerful, and produces less entropy than the MP regime. This follows from the fact that the $\mathrm{M} \Omega$ regime was chosen to represent an optimal compromise between high power output and low entropy production.

(b) The points representing the system state in the phase state occupy a larger area in the case of the $\mathrm{M} \Omega$ regime than in the MP case. This follows from the fact that restitutive forces are weaker in the $\mathrm{M} \Omega$ regime.

(c) Weaker restitutive forces also explain why fluctuations tend to last longer in the thermodynamic time series of the $\mathrm{M} \Omega$ regime.

(d) Finally, the asymmetry of the probability distributions corresponding to the efficiency and entropy production is more pronounced in the M $\Omega$ than in the MP regime. That is, being the engine in the $\mathrm{M} \Omega$ regime it is more likely that it evolves to a more efficient, less entropy-producing state and produces less power due to random perturbations. Interestingly, the asymmetry of the efficiency and entropy-production perturbations does not lead to an asymmetric power-output distribution.

\section{B. Restitutive forces dependent on the values of heat flux into the cold reservoir and power output}

To better understand the dynamics of an LDHE in which restitutive forces depend on the heat fluxes between the engine and the thermal baths, we numerically solved Eqs. (62) and (63). We have considered two different scenarios for the system steady state: maximum power and maximum $\Omega$ function regimes. To do this, we took the stationary values of parameters $\alpha$ and $\tilde{t}$ correspondingly given by Eqs. (60) and (61). The results for the MP regime are shown in Fig. 8, while the $\mathrm{M} \Omega$ regime results are presented in Fig. 9. The parameter values employed in such simulations are $\tau=2 / 5$, $\Sigma_{c}=0.5, c_{v}=0.002$, and $\Delta t=0.05$. In all cases, the initial condition was set to the corresponding steady state.

Observe in Fig. 8 that, in agreement with our former results, the system steady state is metastable in the sense that, in the presence of additive white noise, the system state fluctuates around the steady state for a while, but eventually it diverges to $\alpha \rightarrow 0$, and $\widetilde{t} \rightarrow \infty$. Thus, both the operation time and the contact time with the hot reservoir diverge, meaning that the hot isotherm becomes reversible and all the system irreversibility is concentrated in the cold reservoir. Furthermore, the system thermodynamic variables undergo a similar behavior. That is, they fluctuate around their corresponding steady state for some time, and then diverge. When this happens, the engine efficiency and power output tend to zero, while entropy production tends to infinity, and so the cold isotherm becomes infinitely irreversible.

From Fig. 9 we can appreciate that the system dynamic behavior in the $M \Omega$ regime is qualitatively similar to that of the MP regime. However, there are some interesting differences. In concordance with the thermodynamic properties of the $\mathrm{M} \Omega$ regime, the efficiency is larger than that of the MP 
regime, while the power output and entropy production are smaller. Moreover, the whole dynamics of the $\mathrm{M} \Omega$ regime is slower. For instance, the contact times with thermal baths are longer, as well as the time the system state fluctuates around the steady state before diverging.

\section{CONCLUDING REMARKS}

We have analyzed the local stability of a low-dissipation heat engine under two different dynamics in the MP and $\mathrm{M} \Omega$ regimes. In the first one perturbations on the heat exchanged with thermal baths (independent from each other) are produced by changes in the contact times with the heat reservoirs. A relevant feature is the possibility of linking relaxation times with total operation times, strengthening the idea that optimization and stability are connected.

The study of random perturbations indicates that in the MP regime, even without increments on the power output, the efficiency and entropy generation fluctuate around the stable point with both larger and smaller values with respect to the stable point. On the other hand, for the $\mathrm{M} \Omega$ regime there is a tendency to "prefer" better energetic states in efficiency and entropy generation (larger efficiencies and less entropy generation) but with less power output. Remarkably, although the efficiency and entropy generation probability density functions (PDFs) change their shapes, the mean value of the power output PDF changes as the system operation regime shifts from MP to M $\Omega$, but the PDF preserves its symmetrical shape.

For the second type of dynamics, instabilities are produced by perturbations in the heat exchanged with the cold reservoir and perturbations on power output. The variations on these quantities are achieved by perturbations of the partial contact time with the cold reservoir, $\alpha$, and the total operation time, $\widetilde{t}$. For this dynamics a narrow (in $\widetilde{t}$ ) basin of attraction depending on the $\widetilde{\Sigma}_{c}$ and $\tau$ values is found for both regimes, which we compare in Fig. 5.

The study of random perturbations indicates that these perturbations will eventually carry the system outside the basin of attraction, heading to the nullcline and evolving toward the no-HE region. That is, for the present mechanism, the dependence on the additive noise intensity is an important key to consider in the description of total operation time fluctuations.

The basin of attraction in the $\mathrm{M} \Omega$ regime is narrower than in MP due to more constrained operation conditions, since it is closer to the reversible limit. However, under the same perturbations the system remains on average a longer period of time in the stability basin under M $\Omega$ than in MP conditions as can be noticed by comparing Figs. 8 and 9 .

In summary, the study of the two types of dynamics shows that relaxation times (associated to stability) and operation times (associated to optimized performance regimes) are closely related. Furthermore, random perturbations around the stable point offer relevant energetic information regarding the performance of $\eta, \widetilde{P}$, and $\dot{\widetilde{\Delta S}}$ in the process of relaxation. This gives us insights into the connection between optimization and stability aspects in heat devices.

\section{ACKNOWLEDGMENTS}

I.R.R. acknowledges financial support from EDI-IPNMÉXICO and COFAA-IPN-MÉXICO; M.S. acknowledges financial support from Conacyt-MÉXICO Grant No. FC1132; J.G.A. acknowledges Universidad de Salamanca Contract No. 2017/X005/1 and A.C.H. acknowledges partial financial support from Junta de Castilla y Leon under project SA017P17.
[1] F. Curzon and B. Ahlborn, Efficiency of a Carnot engine at maximum power output, Am. J. Phys. 43, 22 (1975).

[2] F. Angulo-Brown, An ecological optimization criterion for finite-time heat engines, J. Appl. Phys. 69, 7465 (1991).

[3] M. Esposito, R. Kawai, K. Lindenberg, and C. Van den Broeck, Efficiency at Maximum Power of Low-Dissipation Carnot Engines, Phys. Rev. Lett. 105, 150603 (2010).

[4] R. Wang, J. Wang, J. He, and Y. Ma, Efficiency at maximum power of a heat engine working with a two-level atomic system, Phys. Rev. E 87, 042119 (2013).

[5] R. Long, Z. Liu, and W. Liu, Performance optimization of minimally nonlinear irreversible heat engines and refrigerators under a trade-off figure of merit, Phys. Rev. E 89, 062119 (2014).

[6] Z. C. Tu, Stochastic heat engine with the consideration of inertial effects and shortcuts to adiabaticity, Phys. Rev. E 89, 052148 (2014).

[7] V. Singh and R. S. Johal, Feynman's ratchet and pawl with ecological criterion: Optimal performance versus estimation with prior information, Entropy 19, 576 (2017).

[8] I. Iyyappan and M. Ponmurugan, Thermoelectric energy converters under a trade-off figure of merit with broken timereversal symmetry, J. Stat. Mech. (2017) 093207.
[9] Y. Izumida, K. Okuda, J. M. M. Roco, and A. Calvo Hernández, Heat devices in nonlinear irreversible thermodynamics, Phys. Rev. E 91, 052140 (2015).

[10] P. Landsberg and H. Leff, Thermodynamic cycles with nearly universal maximum-work efficiencies, J. Phys. A 22, 4019 (1989).

[11] R. Long and W. Liu, Unified trade-off optimization for general heat devices with nonisothermal processes, Phys. Rev. E 91, 042127 (2015).

[12] R. Long and W. Liu, Ecological optimization for general heat engines, Physica A (Amsterdam, Neth.) 434, 232 (2015).

[13] Y. Zhang, C. Huang, G. Lin, and J. Chen, Universality of efficiency at unified trade-off optimization, Phys. Rev. E 93, 032152 (2016).

[14] B. Cleuren, B. Rutten, and C. Van den Broeck, Universality of efficiency at maximum power: Macroscopic manifestation of microscopic constraints, Eur. Phys. J. Spec. Top. 224, 879 (2015).

[15] V. Holubec and A. Ryabov, Maximum efficiency of lowdissipation heat engines at arbitrary power, J. Stat. Mech. (2016) 073204.

[16] I. Iyyappan and M. Ponmurugan, General relations between the power, efficiency, and dissipation for the irreversible heat 
engines in the nonlinear response regime, Phys. Rev. E 97, 012141 (2018).

[17] M. Esposito, K. Lindenberg, and C. Van den Broeck, Universality of Efficiency at Maximum Power, Phys. Rev. Lett. 102, 130602 (2009).

[18] Z. Ye, Y. Hu, J. He, and J. Wang, Universality of maximumwork efficiency of a cyclic heat engine based on a finite system of ultracold atoms, Sci. Rep. 7, 6289 (2017).

[19] Y. Izumida and K. Okuda, Efficiency at maximum power of minimally nonlinear irreversible heat engines, Europhys. Lett. 97, 10004 (2012).

[20] J. Gonzalez-Ayala, J. M. M. Roco, A. Medina, and A. CalvoHernández, Carnot-like heat engines versus low-dissipation models, Entropy 19, 182 (2017).

[21] R. S. Johal, Heat engines at optimal power: Low-dissipation versus endoreversible model, Phys. Rev. E 96, 012151 (2017).

[22] K. Sekimoto and S. Sasa, Complementarity relation for irreversible process derived from stochastic energetics, J. Phys. Soc. Jpn. 66, 3326 (1997).

[23] T. Schmiedl and U. Seifert, Efficiency at maximum power: An analytically solvable model for stochastic heat engines, Europhys. Lett. 81, 20003 (2008).

[24] C. de Tomas, J. M. M. Roco, A. Calvo Hernández, Y. Wang, and Z. C. Tu, Low-dissipation heat devices: Unified trade-off optimization and bounds, Phys. Rev. E 87, 012105 (2013).

[25] A. Calvo Hernández, A. Medina, and J. M. M. Roco, Time, entropy generation, and optimization in low-dissipation heat devices, New J. Phys. 17, 075011 (2015).

[26] J. Gonzalez-Ayala, A. Calvo Hernández, and J. M. M. Roco, From maximum power to a trade-off optimization of lowdissipation heat engines: Influence of control parameters and the role of entropy generation, Phys. Rev. E 95, 022131 (2017).

[27] P. Pietzonka and U. Seifert, Universal Trade-Off between Power, Efficiency, and Constancy in Steady-State Heat Engines, Phys. Rev. Lett. 120, 190602 (2018).
[28] M. Bauer, K. Brandner, and U. Seifert, Optimal performance of periodically driven, stochastic heat engines under limited control, Phys. Rev. E 93, 042112 (2016).

[29] I. Reyes-Ramírez, J. Gonzalez-Ayala, A. Calvo Hernández, and M. Santillán, Local-stability analysis of a low-dissipation heat engine working at maximum power output, Phys. Rev. E 96, 042128 (2017).

[30] V. Holubec and A. Ryabov, Cycling Tames Power Fluctuations Near Optimum Efficiency, Phys. Rev. Lett. 121, 120601 (2018).

[31] R. Páez-Hernández, F. Angulo-Brown, and M. Santillán, Dynamic robustness and thermodynamic optimization in a non-endoreversible Curzon-Ahlborn engine, J. Non-Equilib. Thermodyn. 31, 173 (2006).

[32] M. Santillán and M. C. Mackey, Dynamic stability versus thermodynamic performance in a simple model for a Brownian motor, Phys. Rev. E 78, 061122 (2008).

[33] O. Díaz-Hernández, R. Páez-Hernández, and M. Santillán, Thermodynamic performance vs. dynamic stability in an enzymatic reaction model, Physica A (Amsterdam, Neth.) 389, 3476 (2010).

[34] Y. Hu, F. Wu, Y. Ma, J. He, J. Wang, A. Calvo Hernández, and J. M. M. Roco, Coefficient of performance for a low-dissipation Carnot-like refrigerator with nonadiabatic dissipation, Phys. Rev. E 88, 062115 (2013).

[35] D. A. Rojas-Gamboa, J. I. Rodríguez, J. Gonzalez-Ayala, and F. Angulo-Brown, Ecological efficiency of finite-time thermodynamics: A molecular dynamics study, Phys. Rev. E 98, 022130 (2018).

[36] J. W. Stuki, Stability analysis of biochemical systems: A practical guide, Prog. Biophys. Mol. Biol. 33, 99 (1978).

[37] M. Santillán, G. Maya, and F. Angulo-Brown, Local stability analysis of an endoreversible Curzon-Ahborn-Novikov engine working in a maximum-power-like regime, J. Phys. D: Appl. Phys. 34, 2068 (2001). 